\title{
Measurements of the intensity noise of a broadly tunable, erbium-doped fiber ring laser, relative to the standard quantum limit
}

\author{
Steve Sanders, Jay W. Dawson, Namkyoo Park, and Kerry J. Vahala \\ Department of Applied Physics, 128-95 California Institute of Technology, Pasadena, California 91125
}

(Received 6 February 1992; accepted for publication 16 March 1992)

The intensity noise of an erbium-doped fiber ring laser is measured relative to the standard
quantum limit. Over a tuning range of $24 \mathrm{~nm}$, the noise power is within $20 \mathrm{~dB}$ of the shot noise
floor and varies linearly with laser output power. Oscillations in the noise power spectrum are
observed and attributed to beating of the lasing mode with other, strongly damped cavity modes.

Due to its broad tunability and single-frequency operation, the tandem fiber Fabry-Perot erbium-doped fiber laser has recently emerged as a potential candidate for communication systems operating at $1.5 \mu \mathrm{m} .^{1,2}$ With the low relaxation resonance frequency of the $\mathrm{Er}^{3+}$ population inversion, this source should have low intensity noise levels at the much higher frequencies that would be used in many applications. In this letter, we present the first measurements of the intensity noise levels in this laser and calibrate these measurements relative to the standard quantum limit (SQL). It is found that the laser operates with the noise power linearly dependent upon the laser power, and at a level within $20 \mathrm{~dB}$ of the SQL throughout a $24 \mathrm{~nm}$ tuning range.

The fiber laser configuration is similar to that described in Ref. 1 and is shown, along with the balanced homodyne detection system used for the intensity noise measurements, in Fig. 1. Optical gain is provided by a Corning FiberGain module which has 20 meters of $\mathrm{Er}^{3+}$ doped fiber, codoped with aluminum. A broadband ( $38 \mathrm{GHz}$ ) fiber Fabry-Perot filter is electrically tunable and selects the lasing wavelength, while a narrow band (130 $\mathrm{MHz})$ fiber Fabry-Perot filter suppresses mode hopping. Single frequency broad band tunability $>30 \mathrm{~nm}$ with sidemode suppression exceeding $48 \mathrm{~dB}$ has been demonstrated for a similar laser. ${ }^{1}$ Measurements of noise power versus optical power were performed by adjusting the current to the InGaAs/GaAs laser pumping the gain medium. At some pumping levels, the laser became unstable, requiring small adjustments (less than $0.2 \mathrm{~V}$, corresponding to less than a $0.2 \mathrm{~nm}$ wavelength shift) to the tunable fiber Fabry-Perot to stabilize the lasing mode.

The balanced homodyne system measures the SQL when the two amplified photocurrents from detectors D1 and D2 are coherently subtracted and the laser noise power when the two photocurrents are coherently summed in a hybrid junction. ${ }^{3}$ Balancing is performed by injecting the light from a $1.5 \mu \mathrm{m}$ distributed feedback semiconductor laser modulated at $310 \mathrm{MHz}$ into the unused port of the fiber laser output coupler. The optical intensities reaching the two detectors (BT\&D PDH0004) are adjusted by rotating a half-wave plate before a polarization sensitive beamsplitter, and the path lengths of the two arms are adjusted to give a common-mode rejection of $>50 \mathrm{~dB}$. A polarizer before the balanced homodyne system maintains the intensity balance when the fiber laser output is measured, and an external polarization controller is optimized before each measurement to ensure that maximum power is transmitted to the measurement system. The shot noise floor is confirmed by retracing it with the laser output power fixed and externally attenuated.

The noise power versus laser power at a fixed wavelength is shown in Fig. 2, along with the shot noise floor. The noise power varies linearly with the output power, as is expected for noise arising from beating between the lasing mode and amplified spontaneous emission in the strongly suppressed side modes. Because of the long cavity length, the free-spectral range is less than $4 \mathrm{MHz}$, so that intensity noise at frequencies far above the relaxation resonance of the fiber laser may be dominated by intermodal beat noise. The relaxation resonance frequency is estimated from a small signal analysis of spatially averaged rate equations ${ }^{4}$ to be on the order of $10 \mathrm{kHz}$.

The structure of the noise spectrum is strongly dependent upon adjustment of the intracavity polarization controller, possibly due to the dependence of the narrow band fiber Fabry-Perot finesse on polarization. In Fig. 3, a 50 MHz span of this spectrum is shown when the polarization controller is adjusted for either flattened or sharply peaked spectra. The flattened spectrum has a variation of less than $5 \mathrm{~dB}$, while the peaked spectrum varies more than $10 \mathrm{~dB}$ over a free-spectral range. However, the minimum noise level in the peaked spectrum is lower than the minimum of the flattened spectrum.

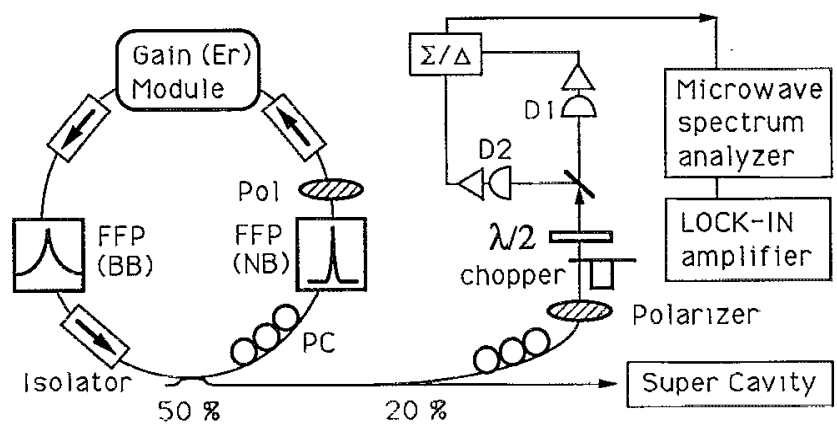

FIG. 1. Ring laser configuration and balanced homodyne detection system (FFP: fiber Fabry-Perot filter, PC: polarization controller). 


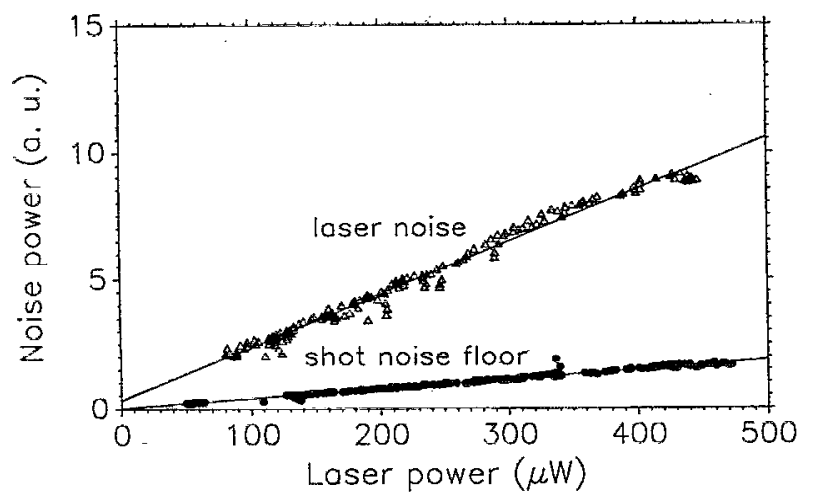

FIG. 2. Measurement of laser noise power at $311 \mathrm{MHz}$ and standard quantum limit vs laser output power after polarizer.

The dependence of the noise power on lasing wavelength is shown in Fig. 4 for the flattened and peaked conditions. The data are normalized to the SQL, which correspond to $0 \mathrm{~dB}$. The solid points represent the maxi mum noise power measured in a free-spectral range around $310 \mathrm{MHz}$, and the open points represent the minimum level. For the flattened spectrum, the peak noise level is 15 $\mathrm{dB}$ above the $\mathrm{SQL}$, and the largest variations throughout the tuning range are $4 \mathrm{~dB}$ for the minima, $4 \mathrm{~dB}$ for the maxima, and $6 \mathrm{~dB}$ overall. A correction for losses of $56 \%$ between the output of the laser ring and the detectors, and
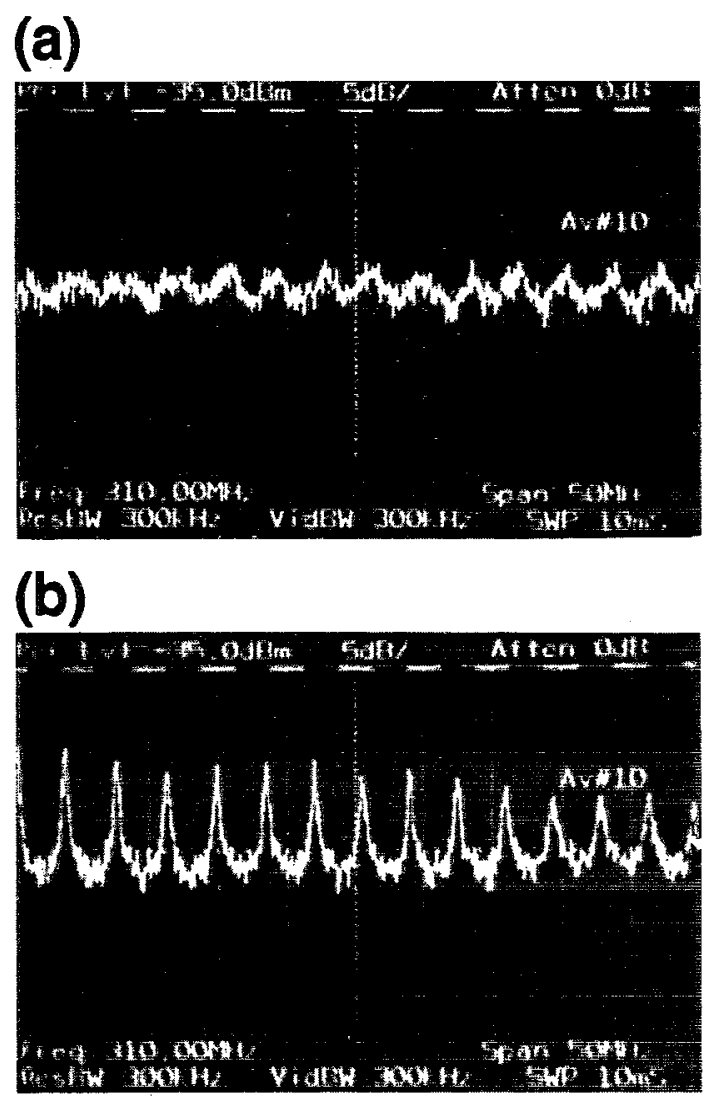

FIG. 3. Laser noise power spectrum with polarization controller adjusted for: (a) flattened condition and (b) peaked condition (resolution bandwidth $=300 \mathrm{kHz}$ ).
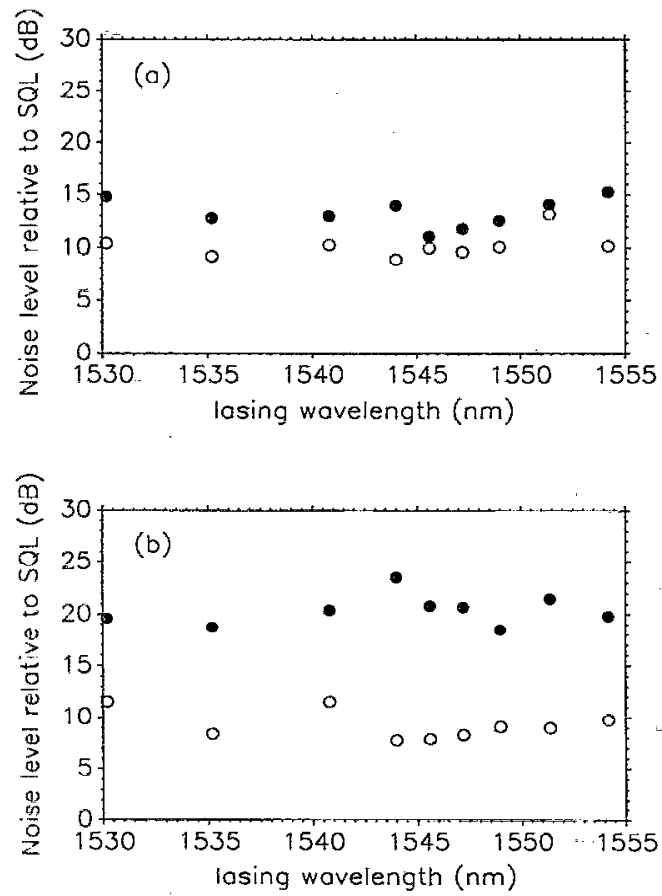

FIG. 4. Laser noise power around $310 \mathrm{MHz}$, measured with $300 \mathrm{kHz}$ resolution, relative to the standard quantum limit vs wavelength for (a) flattened spectrum and (b) peaked spectrum; the solid points represent the maximum noise power and the open points the minimum noise power in one free-spectral range.

for the $80 \%$ detector quantum efficiencies is given by:

$$
W_{\mathrm{obs}}=\eta W+1-\eta
$$

where $W_{\text {obs }}$ is the observed noise power normalized to the SQL; $\eta$ is the overall efficiency, and $W$ is the actual noise power normalized to the SQL. ${ }^{5}$ Over the noise levels measured, this correction requires adding $4.1-4.5 \mathrm{~dB}$ to the data shown. In the flattened spectrum case, this gives a noise level less than $20 \mathrm{~dB}$ relative to the SQL throughout the $24 \mathrm{~nm}$ tuning range measured. Lower noise levels can be obtained by operating the laser in the peaked spectrum condition, but at the expense of much higher peak noise levels within a free spectral range.

We have presented the first intensity noise measurements of a tandem fiber Fabry-Perot erbium laser, demonstrating that the noise power varies linearly with output power and can be within $20 \mathrm{~dB}$ of the SQL over a $24 \mathrm{~nm}$ tuning range. It would be expected that the intensity noise could be reduced by optimization of the gain medium length to reduce the overall level of spontaneous emission and by better suppression of side modes.

Note added in proof: We have measured that the relaxation resonance frequency varies from $10 \mathrm{kHz}$ to $30 \mathrm{kHz}$, increasing as the square root of the laser power. ${ }^{6}$

The authors would like to thank the Office of Naval Research, Contract No. N00014-91-J-1524 and Northrop Corporation for their support of this work.

\footnotetext{
${ }^{1}$ N. Park, J. W. Dawson, K. J. Vahala, and C. Miller, Appl. Phys. Lett. 59, 2369 (1991).

${ }^{2}$ J. L. Zyskind, J. W. Sulhoff, Y. Sun, J. Stone, L. W. Stulz, G. T.
} 
Harvey, D. J. DiGiovanni, H. M. Presby, A. Piccirilli, U. Koren, and R. M. Jopson, Electron. Lett. 27, 2148 (1991).

${ }^{3}$ B. L. Schumaker, Opt. Lett. 9, 189 (1984).

${ }^{4}$ C. R. Giles and E. Desurvire, J. Lightwave Technol. 9, 271 (1991).
${ }^{5}$ S. Machida and Y. Yamamoto, IEEE J. Quantum Electron QE-22, 617 (1986).

'J. W. Dawson, S. Sanders, N. Park, and K. J. Vahala, 1992 OSA Meeting, paper C-00726, Sept. 1992, Albuquerque, NM. 